\title{
Traffic feature-based botnet detection scheme emphasizing the importance of long patterns
}

\author{
Yichen An ${ }^{\text {a) }}$, Shuichiro Haruta, Sanghun Choi, and Iwao Sasase \\ Department of Information and Computer Science, Keio University, \\ 3-14-1 Hiyoshi, Kohoku, Yokohama, Kanagawa 223-8522, Japan \\ a) anyichen@sasase.ics.keio.ac.jp
}

\begin{abstract}
In this paper, we propose a traffic feature-based botnet detection scheme emphasizing the importance of long patterns. Since the communication sequences of bots are not easily changed, the long communication patterns of botnets are useful for detection. The proposed scheme emphasizes the long pattern's importance by improving the feature extraction algorithms and giving weights to the long patterns with large occurrences. By the computer simulation with real dataset, we show the effectiveness of our scheme.
\end{abstract}

Keywords: botnet detection, feature extraction algorithms

Classification: Internet

\section{References}

[1] N. Hoque, D. K. Bhattacharyya, and J. K. Kalita, "Botnet in DDoS attacks: Trends and challenges," IEEE Commun. Surveys Tuts., vol. 17, no. 4, pp. 22422270, 2015. DOI:10.1109/COMST.2015.2457491

[2] C. J. Dietrich, C. Rossow, F. C. Freiling, H. Bos, M. van Steen, and N. Pohlmann, "On botnets that use DNS for command and control," IEEE Seventh European Conference on Computer Network Defense, pp. 9-16, 2011. DOI: 10.1109/EC2ND.2011.16

[3] C. Li, W. Jiang, and X. Zou, "Botnet: Survey and case study," IEEE Fourth International Conference on Innovative Computing, Information and Control (ICICIC), 2009. DOI:10.1109/ICICIC.2009.127

[4] C. Livadas, R. Walsh, D. Lapsley, and W. T. Strayer, "Using machine learning techniques to identify botnet traffic," Local Computer Networks (LCN), pp. 967-974, 2006. DOI:10.1109/LCN.2006.322210

[5] Y.-H. Su, A. Rezapour, and W.-G. Tzeng, "The forward-backward string: A new robust feature for botnet detection," IEEE Conference on Dependable and Secure Computing, 2017. DOI:10.1109/DESEC.2017.8073831

[6] E. B. Beigi, H. H. Jazi, N. Stakhanova, and A. A. Ghorbani, "Towards effective feature selection in machine learning-based botnet detection approaches," IEEE Conference on Communications and Network Security, 2014. DOI:10.1109/ CNS.2014.6997492

[7] S. Saad, I. Traore, A. Ghorbani, B. Sayed, D. Zhao, W. Lu, J. Felix, and P. Hakimian, "Detecting P2P botnets through network behavior analysis and 
machine learning," IEEE Ninth Annual International Conference on Privacy, Security and Trust, pp. 174-180, 2011. DOI:10.1109/PST.2011.5971980

\section{Introduction}

Recently, the computer networks are exposed to the crisis of the botnets. The attacks by botnets include spreading spams, DDoS (Distributed Denial of Service) [1], and so on. The botnet consists of two components called bots and C\&C (Command and Control) servers [2]. The C\&C server sends instructions to bots and the bots follow them. According to [3], about $40 \%$ of the 800 million computers connected to the Internet are botnets. For the secure network, botnet detection is imperative. To detect botnets, many approaches have been proposed.

In [4], Livadas et al. propose an approach which focuses on the fact that the botnets and ordinary users have different features such as size of packets and sending rate. However, the features mentioned above can be manipulated by attackers who try to avoid detection. In order to deal with this, $\mathrm{Su}$ et al. propose a scheme which uses the communication sequence as a feature [5]. The main idea behind that scheme is that the communication sequences of bots are not easily changed and represent special feature since they are controlled by programs which are not frequently updated. In that scheme, the communication sequence is tokenized to truncated sequences by $n$-gram. The occurrences of patterns appeared in the truncated sequences are used as a feature vector. The detection accuracy of that scheme is high. However, since the feature value of the previous scheme [5] is normalized by the total number of all patterns' occurrences, the number of occurrences in larger $n$ is less than that of smaller $n$. That is, regardless of the value of $n$, the previous scheme normalizes the feature values by the fixed number of all patterns' occurrences. As a result, the values of normalized longer patterns' features become smaller and are hidden by other features.

In this paper, we propose a traffic feature-based botnet detection scheme emphasizing the importance of long patterns. First, we normalize occurrences by the total number of occurrences in each $n$, since the smaller occurrences in larger $n$ are normalized by small sum and the feature becomes more balanced with a larger value. Second, we give weights to the features by calculating ranks of the normalized feature, since when longer pattern's occurrence is very large, it is more useful to detect botnet. By computer simulation, we demonstrate that maximum improvement in our scheme is $12 \%$ compared with the previous scheme.

\section{Previous scheme}

The main idea of the previous scheme is that due to the control by the program, the directional information between bots and $\mathrm{C} \& \mathrm{C}$ servers can be used as a feature. The flow of creating the feature in the previous scheme is shown in Table I. As shown in Table I, the original source of feature is called "forward-backward string". In order to identify the communications from botnet to $\mathrm{C} \& \mathrm{C}$ server (in) and from C\&C server to botnet (out), they are calculated by XOR (exclusive OR) and the 
Table I. The feature creation in previous scheme

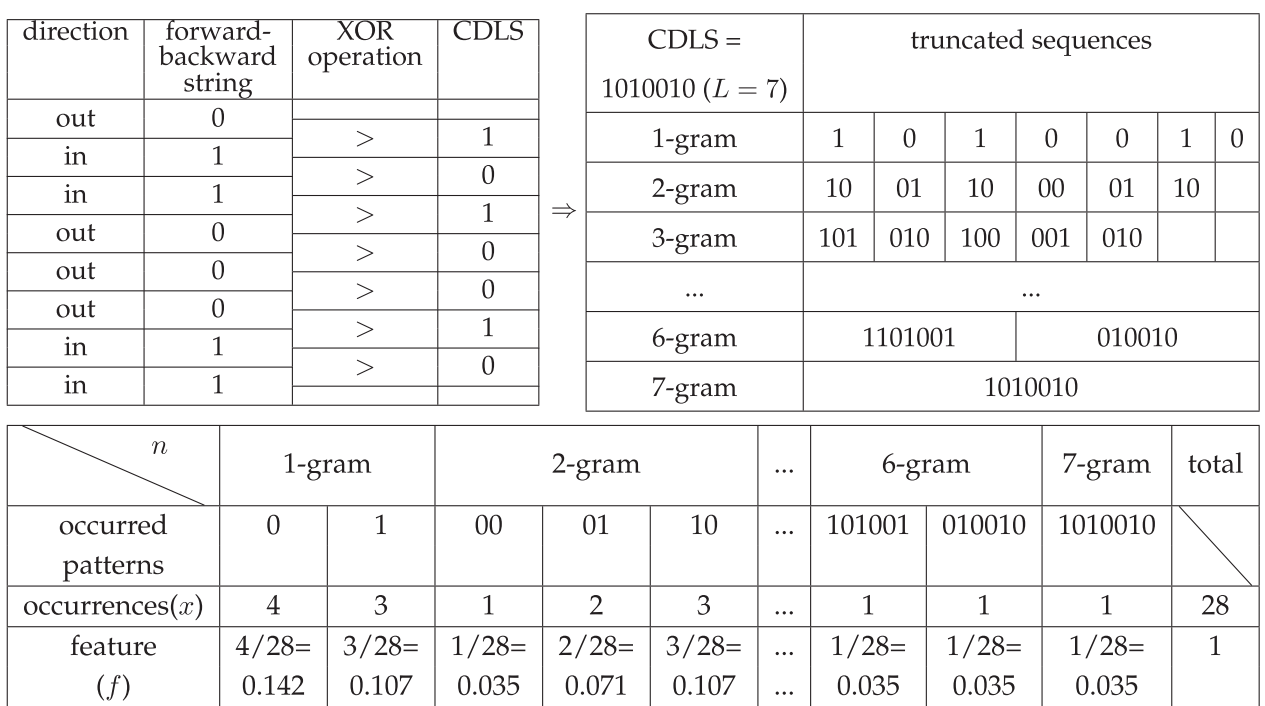

result is called "corresponding direction less string (CDLS)". CDLS is tokenized to truncated sequences by $n$-gram and the occurrences of patterns appeared in the truncated sequences are counted. However, the numbers of occurrences of each pattern are highly dependent on the length of CDLS. In order to mitigate this, these occurrences are normalized by the total number of all patterns' occurrences and they are used as a feature vector.

\subsection{Shortcomings of previous scheme}

Although the feature value of the previous scheme is normalized by the total number of all patterns' occurrences, the number of occurrences in larger $n$ is less than those of smaller $n$. As a result, normalized long patterns' features become very small and are hidden by other features. The bottom table in Table I shows an example of the previous scheme's normalization in the case of CDLS $=1010010$ (the number of digit $L$ is 7). As shown in this table, when $n=7$, the number of occurrence of " 1010010 " is 1 . Although this is small compared to the case $n=1$ where the numbers of occurrences of " 0 " and " 1 " are 4 and 3, respectively, those values are normalized by the same value of 28 , total number of occurrences, in the previous scheme.

\section{Proposed scheme}

We argue the feature in the previous scheme can be improved by emphasizing importance of the long patterns. We realize that emphasizing by two ideas. The first idea is normalizing occurrences by total number of occurrences in each $n$ instead of the number of all patterns' occurrences. By doing this, smaller occurrences in larger $n$ are normalized by smaller value and become more balanced with larger values. The second idea is giving weights to the normalized features according to the importance. In the condition where longer pattern's occurrence is more frequent, it is useful feature for detecting botnet. Thus, we calculate ranks of the normalized features and create new feature according to the ranks. 


\subsection{Normalizing procedure}

Let $x, L, N, f$, and $F$ denote the number of occurrence of the pattern, the length of the CDLS, the maximum $n$ in the $n$-gram, the feature value in the previous scheme, and the modified feature value calculated by normalizing in our proposed scheme, respectively. Since we normalize by total number of occurrences in each $n$, we can describe $F$ as

$$
F=\frac{x}{L-n+1}
$$

on the condition $L \geq N$. Compared with the previous scheme, the value of $F$ is more balanced and larger than the feature $f$ in the previous scheme because the occurrence $x$ is smaller when $n$ is larger. Table II shows the example modified feature in the proposed scheme. By comparing $F$ in Table II with $f$ in Table I, our scheme's features have larger values than the previous ones. In particular, we focus on the patterns where " 1 " and " 10 " have the same occurrence. In this case, while the previous scheme has the same values of $f, F$ of 2-gram is larger than that of 1-gram in our scheme. This indicates that the longer patterns are more emphasized in the proposed normalization.

\subsection{Ranking procedure}

The normalized feature vector is further weighted by ranking procedure. We first calculate ranks for each $n$-gram. The rank of the feature with smallest occurrence is one and that of largest is $2^{n}$. Note that the ranks become the same values if the occurrences are the same and they become one if the occurrence is zero. We weight the feature $F$ by multiplying rank $r$. Let $F^{\prime}$ denote the new feature weighted by ranking procedure. The new feature is represented as

$$
F^{\prime}=F * r=\frac{r x}{L-n+1}
$$

on the conditions $1 \leq r \leq 2^{n}$ and $L>N$. From the former condition, as $n$ is larger, the rank of larger occurrences become larger so that the value of the feature is emphasized. As we can see from Table II, the pattern "10" can be emphasized compared with " 00 " and " 01 " in 2-gram. If $n$ is much larger, more effective emphasizing can be expected.

Table II. Example of modified feature in the proposed scheme

\begin{tabular}{|c|c|c|c|c|c|c|c|c|c|}
\hline \multicolumn{8}{|c|}{ CDLS = 1010010 $(L=7)$} \\
\hline$n$ & \multicolumn{2}{|c|}{1 -gram } & \multicolumn{3}{c|}{ 2-gram } & $\ldots$ & \multicolumn{2}{|c|}{ 6-gram } & 7-gram \\
\hline $\begin{array}{c}\text { occurred } \\
\text { patterns }\end{array}$ & 0 & 1 & 00 & 01 & 10 & $\ldots$ & 101001 & 010010 & 1010010 \\
\hline occurrences $(x)$ & 4 & 3 & 1 & 2 & 3 & $\ldots$ & 1 & 1 & 1 \\
\hline $\begin{array}{c}\text { modified feature } \\
(F)\end{array}$ & $4 / 7=$ & $3 / 7=$ & $1 / 6=$ & $2 / 6=$ & $3 / 6=$ & $\ldots$ & $1 / 2=$ & $1 / 2=$ & $1 / 1=$ \\
\hline rank $(r)$ & 0.571 & 0.428 & 0.166 & 0.333 & 0.5 & $\ldots$ & 0.5 & 0.5 & 1 \\
\hline weighted feature $\left(F^{\prime}\right)$ & 2 & 1 & 2 & 3 & 4 & $\ldots$ & 63 & 63 & 128 \\
\hline
\end{tabular}




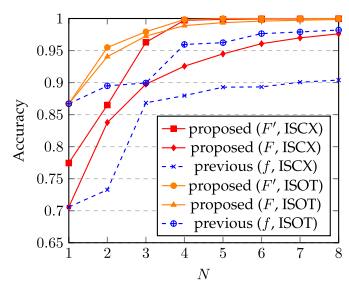

(a) Detection accuracy versus $N$ with $\gamma=10$ in ISOT of ISCX

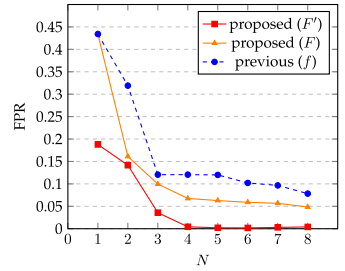

(b) FPR versus $N$ with $\gamma$ $=10$ in ISCX

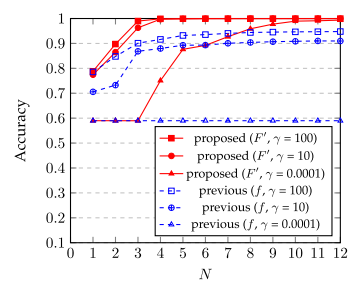

(c) Detection accuracy versus $N$ in ISCX with $\gamma=$ 100,10 and 0.0001

Fig. 1. Detection accuracy of the proposed and previous scheme

\section{Simulation results}

In order to show the effectiveness of the proposed scheme, we evaluate the detection accuracy and FPR (False Positive Rate) calculated as

$$
\text { accuracy }=\frac{T P+T N}{T P+T N+F P+F N}
$$

and

$$
F P R=\frac{F P}{T N+F P},
$$

where TP, TN, FP, and FN denote the number of True Positive, True Negative, False Positive, and False Negative, respectively. Each result is yielded by 10 -fold cross validation. We use SVM (Support Vector Machine) as the machine learning classifier with a parameter $\gamma$, which indicates how far the influence of each training samples reaches. Although the larger value of $\gamma$ brings better result for SVM, that includes the risk of overlearning. In order to prove our idea, we test the effect of this parameter. We use ISCX [6] as a primary dataset and supplementally use ISOT dataset [7].

\subsection{Overall tendency}

Fig. 1(a) shows detection accuracy of the proposed and previous schemes versus $N$ when the dataset ISCX and ISOT are used. The parameter $\gamma$ is fixed to 10. As we can see from Fig. 1(a), the detection accuracy of the previous scheme [5] and the proposed scheme increases as $N$ increases in both of dataset. This is because the number of patterns increases and longer patterns are more valuable. However, in the previous scheme, the increase of detection accuracy is slow. On the other hand, the proposed schemes achieve rapid increase in both of dataset. Especially in ISCX, when $N=4$, our normalizing and ranking procedures improve the performance compared to the previous scheme by $5 \%$ and $12 \%$, respectively. In ISOT, those procedure improvements are by $3 \%$ and $4 \%$, respectively. The reason why there is difference in the performance between two datasets is that ISOT includes fewer types of botnets. Thus, it is relatively easy to classify bots and ordinary users, and as a result, the difference of the accuracy between our schemes is small. 


\subsection{False-positive rate}

Fig. 1(b) shows FPR of the proposed and previous schemes versus $N$ in ISCX dataset. As we can see from Fig. 1(b), FPR of both schemes decrease as $N$ increases. Comparing the proposed and previous scheme, the proposed scheme rapidly decreases FPR and it is close to zero. This is because the longer patterns' features are effectively reflected.

\subsection{Effectiveness of emphasizing longer pattern}

Fig. 1(c) shows detection accuracy of the proposed and previous schemes versus $N$ with multiple $\gamma$ in ISCX dataset. We set the parameter $\gamma$ to 100,10 , and 0.0001 . As we can see from Fig. 1(c), the proposed scheme improves the performance compared to the previous scheme in all $\gamma$ pairs. Focusing on the lines whose $\gamma=0.0001$, the detection accuracy of the proposed scheme approaches 1.0, when $N \geq 4$.

\section{Conclusion}

We have proposed a traffic feature-based botnet detection scheme emphasizing the importance of long patterns by normalizing and ranking procedures. By the computer simulation with real dataset, we show the maximum improvement in our scheme is $12 \%$ compared with the previous scheme.

\section{Acknowledgment}

This work is partly supported by the Grant in Aid for Scientific Research (No. 17K06440) from Japan Society for Promotion of Science (JSPS). 